\title{
The role of human leucocyte antigen genes in the development of malignant disease
}

\author{
W M Howell, D B Jones
}

\begin{abstract}
Introduction
The human leucocyte antigen (HLA) class I and in particular class II genes play a major role in regulating the immune response. The extensive polymorphism of these genes has been characterised by a series of international histocompatibility workshops undertaken during the past 31 years, with the current (Twelfth) workshop due to conclude in St Malo in June 1996. Many studies, both within and without these workshops, have revealed significant associations between particular polymorphisms of the HLA class I and especially class II genes and predisposition to a large number of benign, immune system mediated diseases including rheumatoid arthritis, ${ }^{1}$ coeliac disease, ${ }^{2}$ insulin dependent diabetes, ${ }^{3}$ multiple sclerosis, ${ }^{4}$ Graves's disease, ${ }^{5}$ and $\operatorname{IgE}$ responsiveness to various pollen and animal dander allergens. ${ }^{6}$ HLA class II allelic associations have also been detected in other benign conditions with no obvious immunological component, such as narcolepsy. ${ }^{7}$ However, the first HLA and disease association to be reported in 1967 was between the HLA class I B35, B5 and B18 group of alleles (then known as $4 \mathrm{C}$ ) and a malignant condition-Hodgkin's disease. ${ }^{8}$ Nevertheless, until recent years there has been comparatively little interest in HLA genetic polymorphism and predisposition to malignant diseases. This has now changed, particularly since the advent of accurate, high resolution DNA based methods for analysis of HLA polymorphism. A small but growing literature now exists relating HLA class II polymorphism and predisposition to a number of malignancies. In addition, downregulation of HLA class I expression on tumour cells would seem to be a frequent route by which tumour cells escape from $\mathrm{T}$ cell mediated attack. These more recent findings will be outlined below.
\end{abstract}

\section{The HLA system}

The classic human major histocompatibility complex or HLA molecules are encoded by two highly polymorphic gene families located in a 3600 kilobase region of chromosome $6 \mathrm{p}(6 \mathrm{p} 21 \cdot 3)$. The resulting HLA moleculesthe most polymorphic found in humans-are membrane bound glycoproteins that bind processed antigenic peptides and present them to $T$ cells. The HLA class I A, B and C molecules are each composed of a HLA encoded heavy chain (MW $45 \mathrm{kD}$ ), non-covalently associated with a non-polymorphic polypeptide $\beta_{2}$ microglobulin (MW $12 \mathrm{kD}$ ) encoded on chromosome 15. There are now known to be at least 50 HLA-A, 97 HLA-B and 34 HLA-
C alleles. ${ }^{9}$ These HLA class I antigens are expressed on all nucleated cells (except fetal trophoblast) and platelets and function to present peptides of largely endogenous (viral) origin to CD8 + T cells, which are mostly cytotoxic. The polymorphic residues which distinguish between the different alleles of a particular HLA class I locus are mainly found within the peptide binding groove and thus control the specificity of peptide binding and presentation to $T$ cells.

In contrast to class I molecules, HLA class II molecules, comprising the three main subclasses DR, DQ and DP, are found on a more restricted range of cell types, including $B$ cells, activated $T$ cells and the monocyte/macrophage lineage, and can be induced by interferon- $\gamma$. An expressed class II molecule consists of an $\alpha$ chain (MW $31-34 \mathrm{kD}$ ) encoded by an $\mathrm{A}$ gene, non-covalently associated with a $\beta$ chain (MW 26-29 kD), encoded by a B gene. Each $\mathrm{DR}, \mathrm{DQ}$ and DP subregion consists of at least one expressed $\mathrm{A}$ and $\mathrm{B}$ gene. There are now known to be at least two DRA, $120 \mathrm{DRB}, 15$ DQA, 26 DQB, eight DPA, and 59 DPB alleles. ${ }^{9}$ Both $\alpha$ and $\beta$ chains combine to form a peptide binding groove, shown by $x$ ray crystallography to be very similar to the class I groove ${ }^{10}$ but capable of accommodating longer peptides, of more variable length. Class II molecules, however, present peptides of largely exogenous origin to CD $4+\mathrm{T}$ cells of mainly helper phenotype.

In the triggering of an anti-tumour immune response $T$ cells must recognise specific tumour antigen derived peptides, including virally encoded proteins as presented by HLA class I or II molecules. Because of the extensive polymorphism of the HLA class I and II genes, an individual's ability to present such viral or tumour specific processed peptides will vary and so some individuals may show reduced immune surveillance of specific viral or somatic changes associated with malignant transformation. Accordingly, it might be expected that individual malignancies, and especially those that are virally induced, might be associated with specific HLA alleles. This has been shown to be the case.

\section{Early studies}

Save for the previously mentioned weak association between HLA class I alleles and Hodgkin's disease and before the advent of high resolution DNA based HLA typing methods, few other studies provided evidence for a link between particular HLA alleles and malignant diseases. One notable exception was 
a study utilising cellular primed lymphocyte typing (detecting limited polymorphism at the HLA-DP loci) which demonstrated an association between the DPw2 (DPB1*02) and DPw5 (DPB1*05) alleles and acute lymphocytic leukaemia. ${ }^{11}$ More recently, a DNA based study has also provided evidence of a weak association between HLA-DPB1*0201 and childhood common acute lymphoblastic leukaemia, ${ }^{12}$ an association, according to the authors, suggestive of an infectious aetiology for this disease, although this remains speculative.

\section{Hodgkin's disease}

Following the early HLA class I studies in Hodgkin's disease, HLA class II DR alleles were investigated as serological typing reagents became available; however, no significant associations were seen. The advent of improved DNA based methods for HLA-DP typing lead to investigations of the contribution of HLADP to susceptibility to this malignancy and an initial restriction fragment length polymorphism (RFLP) based study showed a significant decrease in the frequency of a DPw2 associated RFLP in patients with Hodgkin's disease. There was also a non-significant increase in a DPw3, 5 and 6 associated RFLP in this report. ${ }^{13}$ These findings were investigated systematically in caucasian and non-caucasian ethnic groups using much higher resolution PCR oligotyping in the Eleventh International Histocompatibility Workshop. Results indicated that in the combined caucasian groups studied, DPB 1*0301 conferred a small, but significant, increased risk of Hodgkin's disease ( $R R=1.95)$, while in orientals DPB1*0401 conferred a significant protective effect $(R R=$ $0 \cdot 148) .^{14}$ In addition, in Japanese patients, DPB $1 * 0901$ was associated with a shorter overall duration of remission. ${ }^{14}$ Thus, Hodgkin's disease is more strongly associated with HLADP than with any other HLA gene examined to date, although the actual suceptibility gene may not be within the HLA-DP region itself. However, an association with HLA may support a viral aetiology for the disease, with viral protein derived peptides being differentially presented to $\mathrm{T}$ cells by particular HLA polymorphic specificities.

\section{Squamous cell cervical carcinoma and} cervical intraepithelial neoplasia

One further area in which there has been much interest in recent years is the possible involvement of HLA in the predisposition to squamous cell carcinoma of the cervix. In the past decade there has been growing evidence that certain subtypes of human papilloma virus (HPV) $(16,18,31$, and 33) are implicated in the development of high grade cervical intraepithelial neoplasia (CIN) and invasive cervical cancer. ${ }^{15-17}$ However, epidemiological studies have also shown that HPV infection in itself, regardless of subtype, is not sufficient to induce cervical cancer. One such additional factor may be impairment of the immune response to particular HPV antigens. Consistent with this model is the finding in some, but not all, studies that particular HLA class II DQB1 alleles are associated with an increased relative risk of squamous cell carcinoma of the cervix in several populations, including European caucasians and Tanzanian and African American women. ${ }^{18-24}$ In German caucasian patients, high resolution PCR oligotyping has revealed that $\mathrm{DQB1}{ }^{*} 0301$ and 0303 are the primary risk alleles for squamous cell cervical cancer. ${ }^{21}$ Similar high resolution typing in African Americans $^{22}$ has demonstrated that of the DQB1*03 allele family, only DQB $1 * 0303$ (along with DQB1*0604) is a risk allele, whilst in Tanzanian patients the HLA association is with DQB1*0602. ${ }^{21}$ These epidemiological differences may reflect ethnic variation in HLA class II allele frequencies and linkage disequilibria between the populations studied or differing criteria for patient recruitment into the various studies.

In addition to the documented HLA class II DQB1 genetic associations with carcinoma of the cervix, some studies have reported that HLA-DQB $1 * 03$ associated alleles may predispose to CIN. ${ }^{25}{ }^{26}$ Furthermore, a significant association has been demonstrated between particular HPV subtypes (HPV 16 and 18) and the presence of the DQB1*0602 allele. $^{2127}$ In one of these studies ${ }^{21}$ patients who had the best prognosis were negative for the three squamous cell carcinoma associated DQB1*0602, 0301 and 0303 alleles.

The HLA class I B*07 allele may also be associated with poorer clinical outcome in cervical cancer and in HPV 16 infected HLA$\mathrm{B}^{*} 07$ individuals, a consistent variation in the HPV 16 E6 oncoprotein sequence has been identified, which alters the HLA-B*07 binding epitope in a way likely to influence immune recognition by cytotoxic $\mathrm{T}$ lymphocytes. ${ }^{28}$ Thus, HLA genotype, HPV strain and genetic variation within that strain may be involved in predisposition to cervical carcinoma or progression from low grade CIN. However, the association between HLA alleles and susceptibility to infection by subtypes of HPV and the interaction between these risk factors in the development of CIN and squamous cell carcinoma of the cervix have yet to be determined.

\section{Other virus associated malignancies}

HLA associated susceptibility to several other forms of virus related malignancies has also been described, including HLA-DR5 and $\mathrm{Ka}$ posi sarcoma, ${ }^{29}$ HLA-A1, B12 and DR7 with Burkitt lymphoma, ${ }^{30}$ and an association among HTLV-1 infection, adult $T$ cell leukaemia and HLA has been suggested. ${ }^{31}$ In addition, the occurrence of skin cancer in the normal population has been shown to be associated with HLA-DR $1^{32-34}$ and with HLA-DR7 in renal transplant recipients. ${ }^{356}$ This difference may reflect a different aetiology of skin cancer in renal transplant recipients, when immunosuppressive therapy renders the patients susceptible to frequent infections with HPVs, 
although a causal role of HPV in the development of skin cancer has yet to be confirmed.

\section{Cutaneous melanoma}

There is evidence that HLA class II alleles contribute to the immune response against the tumour in cutaneous melanoma. While earlier studies based upon serological typing have variably suggested that there is an association between HLA-DR and susceptibility to melanoma, ${ }^{3738}$ a recent study using PCR oligotyping has demonstrated a highly significant association between cutaneous melanoma and HLA-DQB $1{ }^{*} 0301$ in American caucasians. ${ }^{39}$ Furthermore, the presence of DQB $1 * 0301$ in these patients was also associated with more advanced disease, such as thicker primary tumours and a higher occurrence of local or distant metastatic spread. ${ }^{39}$ The mechanism for this HLA-DQB1 association with melanoma remains unclear. However, it is possible that the $\mathrm{DQB} 1{ }^{*} 0301$ allele regulates the immune response to melanoma through lack of expression of HLA-DQ on the tumour or effector cell surface, with the resulting lack of presentation of class II associated tumour specific peptides to $\mathrm{T}$ cells. Expression of the DQB1*0301 allele could also stimulate CD4 suppressor $\mathrm{T}$ cells, suppressing an effective immune response to melanoma. There may also be a gene linked to regulating the antimelanoma immune response and linked to HLA-DQB.

\section{Coeliac disease and enteropathy associated $T$ cell lymphoma}

With few exceptions, there has been little investigation of possible HLA genetic associations which might contribute to the development of lymphoma. Some recent results from our laboratory concerning the relation between coeliac disease and enteropathy associated $T$ cell lymphoma are therefore of interest because they suggest that HLA class II genetic polymorphism not only predisposes to coeliac disease, but also influences the development of malignancy within this benign enteropathy.

Coeliac disease is an immune mediated disease of the small intestinal mucosa characterised by flattening of the small intestinal villi and non-specific malabsorption, elicited by the ingestion of gluten, a protein found in several cereals, including wheat, rye, barley, and oats. While it had been known for some time that susceptibility to coeliac disease is associated with particular HLA class II polymorphisms, the extensive studies performed in the Eleventh International Histocompatibility Workshop confirmed that susceptibility to coeliac disease is primarily associated with expression of a particular HLA-DQ heterodimer (HLA-DQ $[\alpha 1 * 0501, \beta 1 * 0201]){ }^{2}$ The alleles encoding this heterodimer may be located in cis or trans configuration. The precise genetic mechanism involved in disease susceptibility is not clear; however, it has been shown that activated $T$ cells derived from small bowel lamina propria biopsy specimens taken from DQA $1 * 0501$, DQB $1 * 0201$ patients with coeliac disease can recognise $\alpha$-gliadin (gluten) derived peptides when presented by cells bearing the DQA $1 * 0501$, DQB1*0201 heterodimer, encoded in cis or trans. This demonstrates that HLA-DQ molecules can effectively present antigenic peptides to $T$ cells in the target organ. ${ }^{40}$ Patients with coeliac disease are at a greater risk of developing malignancy than the general population. These tumours are principally malignant lymphomas, including those of gastrointestinal origin. The latter are of a high grade, have a $\mathrm{T}$ cell genotype and have been designated enteropathy associated $\mathrm{T}$ cell lymphoma. ${ }^{41}$ However, controversy reigns over whether enteropathy associated $\mathrm{T}$ cell lymphoma arises in pre-existing coeliac disease or is in fact a distinct entity, with a precursor stage of low grade lymphoma, masquerading as coeliac disease. ${ }^{4243}$

In order to investigate the genetic relation between coeliac disease and enteropathy associated $\mathrm{T}$ cell lymphoma and to distinguish between these conflicting models of the origin of malignant enteropathy, we studied HLA class II DRB1, DQA1 and DQB1 polymorphism in patients with uncomplicated coeliac disease and enteropathy associated $\mathrm{T}$ cell lymphoma, all of UK caucasoid origin, using PCR oligotyping of paraffin wax biopsy specimen derived DNA. ${ }^{44}$ Our results demonstrated highly significant increases in frequencies of DRB $1 * 03$ and DQA $1 * 0501, \mathrm{DQB} 1 * 0201$ genotypes both the patients with uncomplicated coeliac disease and in those with enteropathy associated $T$ cell lymphoma when compared with controls. These data suggested that enteropathy associated $\mathrm{T}$ cell lymphoma arises in the same genetic background as coeliac disease. However, some differences between the two patient groups were also observed. In addition to an increase in DRB1*0304 heterozygosity in the patients with enteropathy associated $\mathrm{T}$ cell lymphoma and an increase in DRB1*0307 heterozygosity in those with coeliac disease, the most notable difference was an absence of DQB1*0201 homozygosity in the patients with enteropathy associated $\mathrm{T}$ cell lymphoma compared with a highly significant increase in DQB1*0201 homozygosity in those with coeliac disease. The genotypes of patients with late onset coeliac disease also resembled those of patients with enteropathy associated $\mathrm{T}$ cell lymphoma more closely than those of patients with early onset coeliac disease. Considering these and other studies of DQB 1 alleles in coeliac disease, ${ }^{4546}$ it can be postulated that in DQA1*0501, DQB1*0201 individuals, the presence of a second DQB $1 * 0201$ allele (along with other HLA or non-HLA genetic influences) predisposes to earlier onset or more severe coeliac disease, leading to earlier diagnosis and treatment. However, non-HLADQB1*0201 homozygous individuals may show sub-clinical symptoms, which if left undetected and untreated, could result in the evolution of neoplastic $T$ cell clones from polyclonal $T$ cell populations in the enteropathic bowel. Thus, the continuing antigen drive (de- 
rived from wheat gluten) in coeliac disease may be a prerequisite for the subsequent development of lymphoma. Paradoxically, in those patients with HLA class II DQB1 (and possibly DRB1) genotypes, which predispose to more severe symptoms in coeliac disease, this antigen drive may indirectly provide protection against the subsequent development of lymphoma.

\section{Colorectal carcinoma}

In adenocarcinoma of the colon a 13Asp mutation in the K-ras oncogene constitutes $25-30 \%$ of all K-ras mutations found in colorectal carcinomas. One recent study showed that this mutation was recognised by HLA-DQB 1 *0301 restricted $\mathrm{T}$ cells in a patient with colorectal carcinoma. ${ }^{47}$ In addition, the same study showed that in a large series of patients with colorectal cancer, the presence of the DQB $1{ }^{*} 0301$ allele seemed to have a modifying effect on the development of the carcinoma, with fewer tumours reaching advanced Dukes' stages when the DQB1*0301 allele was present. ${ }^{47}$ This would appear to be a clear-cut example of a particular HLA specificity playing a protective role against malignancy, by a presentation of a tumour associated antigenic peptide to host $\mathrm{T}$ cells.

\section{Defective immune surveillance in malignancy}

While the above examples are not exhaustive, they have been chosen to illustrate specific aspects of some of the mechanisms by which particular HLA polymorphisms may predispose to or protect against the development of malignancy. However, as expression of HLA class I molecules normally occurs on virtually all nucleated cells, one method by which tumour cells could avoid a CD8 cytotoxic T cell response would be via deficient HLA class I expression. Again, clearly documented examples exist. In Hodgkin's disease, while ReedSternberg cells strongly express HLA class II antigens, a recent study has shown that expression of class I antigens is absent ${ }^{48}$ providing an explanation for the lack of a CD8 $\mathrm{T}$ cell response against Reed-Sternberg cells in this disease. The study which provided evidence that HLA-B*07 was associated with a poorer clinical outcome in cervical carcinoma also demonstrated a loss of HLA-B*07 expression in the cervical tumour cells (reviewed in ${ }^{28}$ ). Loss of HLA-B*07/B*40 expression has been shown to be highly correlated with the metastatic spread of cervical neoplasia. ${ }^{28}$ In addition, HLA class I expression is frequently absent or reduced in colorectal carcinoma cell lines. In some cases the genetic defect leading to absence of class I antigen expression would seem to be in the $\beta_{2}$-microglobulin gene. ${ }^{49}$ This absence of $\beta_{2}$-microglobulin expression correlates with a "mutator" phenotype, leading to a DNA mismatch correction defect. These data suggest therefore that "mutator" tumour cells have undergone selection to evade $T$ cell surveillance via loss of HLA class I expression.

\section{Conclusions}

While the bulk of HLA and disease studies have concentrated on benign, immune mediated conditions, the growing body of literature now supports a priori expectations that the extensive HLA class I and II polymorphism should lead to variations in antitumour surveillance between individuals. Clear examples indicate that particular HLA alleles or haplotypes predispose to the development of specific malignancies. As viral aetiologies and tumour specific mutations are uncovered in more malignancies, the basis for some of the reported HLA associations with malignancy will become clearer. The widespread application of high resolution PCR based methods for HLA typing will increase the scope for further studies, particularly when such methods can be applied to DNA derived from stored, paraffin wax embedded biopsy specimens. Studies of HLA expression in malignancy will also yield further examples of tumours evading $\mathrm{T}$ cell immune surveillance by downregulation or absence of HLA class I antigen expression. Such malignancies, if also shown to have a mutator phenotype, may present particular therapeutic problems. ${ }^{49}$ Despite this, studies of HLA polymorphism in malignant disease, together with the identification of specific tumour associated antigen HLA binding epitopes should ultimately provide a new approach for the design of appropriate anti-tumour vaccines.

1 Winchester R. The molecular basis of susceptibility to rheumatoid arthritis. Adv Immunol 1994;56:389-466.

2 Hall MA, Mazzilli MC, Satz ML, Barboni F, Bartova A, Brunnler G, et al. Coeliac disease study. In: Tsuji K, Aizawa M, Sasazuki T, eds. HLA 1991. Proceedings of the Eleventh International Histocompatibility Workshop and the Eleventh International Histocompatibility Workshop and Conference.

3 Thorsby E, Ronningen KS. Role of HLA genes in predisposition to develop insulin-dependent diabetes mellitus. Ann Med 1992;24:523-31.

4 Oksenberg JR, Begovich AB, Erlich HA, Steinman L. Genetic factors in multiple sclerosis. $\mathcal{F} A M A$ 1993;270:2362-9.

5 Boehm BO, Thomas H, Lee J, Chan SH, Cheah JS, Nishimura Y, et al. Graves's disease study. In: Tsuji K, Aizawa M Sasazuki T, eds. HLA 1991 Proceedings of Aizawa M, Sasazuki T, eds. HLA 1991. Proceedings of the Eleventh International Histocompatibility Workshop and Conference. Vol 1. Oxford: Oxford University Press, 1992: 710-13.

6 Howell WM. HLA immunogenetics and specific IgE responses to allergens. Clin Exp Immunol 1994;24:401-4.

7 Mignot E, Lin X, Arrigoni J, Macaubas C, Olive F, Hallmayer J, et al. DQB1*0602 and DQA ${ }^{*} 0102$ (DQ1) are better markers than DR2 for narcolepsy in Caucasian and black Americans. Sleep 1994;17:S60-7.

8 Amiel JL. Study of the leukocyte phenotypes in Hodgkin's disease. In: Curtoni ES, Mattiuz PL, Tosi RM, eds. Histocompatibility testing. Munksgaard, Copenhagen: 1970 $79-81$.

9 Bodmer JG, Marsh SGE, Albert ED, Bodmer WF, Dupont B, Erlich HA, et al. Nomenclature for factors of the HLA B, Erlich HA, et al. Nomenclature for factor
system, 1994. Tissue Antigens 1994;44:1-18

10 Brown JH, Jardetsky TS, Gorga JC, Stern LJ, Urban RG, Strominger JL, Wiley DC. Three-dimensional structure of the human class II histocompatibility antigen HLADR1. Nature 1993;364:33-9.

11 Pawelec G, Ehninger G, Muller C, Blaurock M, Schneider $E M$, Wernet $P$. Human leukocyte antigen-DP in leukemia Cancer 1988;61:475-7.

12 Taylor GM, Robinson MD, Binchy A, Birch JM, Stevens RF, Jones PM, et al. Preliminary evidence of an association between HLA-DPB 1*0201 and childhood common acute between HLA-DPB 0201 and childhood common acute Leukemia 1995;9:440-3.

13 Bodmer JG, Tonks S, Oza AM, Lister TA, Bodmer WF HLA-DP based resistance to Hodgkin's disease. Lancet HLA-DP based

14 Bodmer JG, Tonks S, Oza AM, Mikata A, Takenouchi T Lister TA and collaborating centres. Hodgkin's disease Lister TA and collaborating centres. Hodgkin's disease
study. In: Tsuji K, Aizawa M, Sasazaki T, eds. HLA 1991. Proceedings of the Eleventh International Histocompatibility Proceedings of the Eleventh International Histocompatibility
Workshop and Conference. Vol 1. Oxford: Oxford University Workshop and Conference
Press, 1992:701-9. 
15 Munoz N. A causal link between HPV and invasive cervical cancer. Int $\mathcal{f}$ Cancer 1992;52:743-9.

16 Nuovo GJ, Blanco JS, Leipzig S, Smith D. HPV detection in cervical lesions non-diagnostic for CIN. Obstet Gynecol 1990;75:1006-11

17 Cuzick J, Terry G, Ho L, Hollingworth T, Anderson M. Type specific HPV DNA in abnormal smears as a predictor of high grade CIN. Br f Cancer 1994;69:167-70.

18 Wank R, Thomssen C. High risk of squamous cell carcinoma of the cervix for women with HLA-DQw3. Nature 1991; 352:723-5.

19 Wank R, Schendel DJ, Thomssen C. HLA antigens and cervical carcinoma. Nature 1992;356:22-3.

20 Helland A, Borresen AL, Kaern J, Ronningen KS, Thorsby E. HLA antigens and cervical carcinoma. Nature 1992; 356:22.

21 Wank R, Meulen JT, Luande J, Eberhardt H-C, Pawlita M. Cervical intraepithelial neoplasia, cervical carcinoma and risk for patients with HLA-DQB $1 * 0602,{ }^{*} 0301$ and $* 0303$ risk for patients with HLA-DQB
alleles. Lancet 1993;341:1215.

22 Gregoire L, Lawrence WD, Kukuruga D, Eisenbrey AB Lancaster WD. Association between HLA-DQB1 alleles and risk for cervical cancer in African-American women. Int $\mathcal{F}$ Cancer 1994;57:504-7.

23 Glew SS, Duggan-Keen M, Ghosh AK, Ivinson A, Sinnott $P$, Davidson J et al. Lack of association of HLA polymorphisms with human papillomavirus-related cervical cancer. Hum Immunol 1993;37:157-64.

24 Aldener A, Rosenlund B, Bistoletti P, Olerup O. No association with $\mathrm{DQ} 3$ or $\mathrm{DQB1}^{*} 03$ alleles in Swedish patients with squamous cell carcinoma of the cervix uteri. Hum Immunol 1993;36:49.

25 David ALM, Taylor GM, Gokhale D, Aplin JD, Seif MW Tindall VR. HLA-DQB ${ }^{*} 03$ and cervical intraepithelial neoplasia type III. Lancet 1992;340:52.

26 Vandenvelde C, DeFoor M, Van Beers D. HLA-DQB $1 * 03$ and cervical intraepithelial neoplasia grade I-III. Lancet 1993;341:442-3.

27 Apple RJ, Erlich HA, Klitz W, Manos MM, Becker TM, Wheeler CM. HLA-DR-DQ associations with cervical
carcinoma show papillomavirus-type specificity. Nature carcinoma show papil
Genet 1994;6:157-62.

28 Ellis JRM, Keating PJ, Baird J, Hounsell EF, Renouf DV, Rowe M, et al. The association of an HPV16 oncogene variant with HLA-B7 has implications for vaccine design in cervical cancer. Nature Med 1995;1:464-70.

29 Scorza Smeraldi R, Fabio G, Lazzarin A, Eisera NB, Moroni $M$, Zanussi C. HLA-associated susceptibility to acquired immunodeficiency syndrome in Italian patients with human-immunodeficiency-virus infection. Lancet 1986;ii $1187-9$.

30 Jones EH, Biggar RJ, Nkrumah FK, Lawler SD. Study of the HLA system in Burkitt's lymphoma. Hum Immunol 1980;1:207-10.

31 The T- and B-cell Malignancy Study Group: The third nationwide study on adult T-cell leukaemia/lymphoma (ATL) in Japan: characteristic pattern of HLA antigen and HTLV-1 infection in ATL patients and their relatives. and HTLV-1 infection in ATL

32 Cerimele D, Contu L, Carcassi C, Costa G, La Nasa G, Sanna E, Campus GV. HLA and multiple skin carcinomas. Dermatologica 1988;176:176-81.
33 Czarnecki D, Lewis A, Nicholson I, Tait B. Multiple basal cell carcinomas and HLA frequencies in southern Australia. F Am Acad Dermatol 1991;24:559-61.

34 Czarnecki D, Lewis A, Nicholson I, Tait B. HLA-DRl is not a sign of poor prognosis for the development of multiple basal cell carcinomas. 7 Am Acad Dermatol 1992; 26:717-19.

35 Czarnecki D, Zalcberg J, Nicholson I, Tait B. Skin cancer and HLA antigens. N Engl f Med 1992;326:765-6.

36 Czarnecki D, Watkins F, Leahy S, Dyall-Smith D, Levis A, Nicholson I, Tait B. Skin cancers and HLA frequencies in renal transplant recipients. Dermatology 1992;185:9.

37 Barger BO, Acton RT, Soong SJ, Roseman J, Balch C. Increase of HLA-DR4 in melanoma patients from Alabama. Cancer Res 1982;42:4276-9.

38 Mueller-Eckhardt G, Schendel DJ, Hundeiker M, Riedel T, O'Neil GJ, Riethmuller G, Mueller-Eckhardt C. Possible association between HLA-DR5 and superficial spreading melanoma (SSM). Int $\mathcal{F}$ Cancer 1984;34:751-5.

39 Lee JE, Reveille JD, Ross MI, Platsoucas CD. HLADOB1*0301 association with increased cutaneous melanoma risk. Int $\mathcal{f}$ Cancer 1994;59:510-13.

40 Lundin KEA, Gjertsen HA, Scott H, Sollid LM, Thorsby E. Function of DQ2 and DQ8 as HLA susceptibility molecules in celiac disease. Hum Immunol 1994;41:24-7.

41 Isaacson PG, O'Connor NJT, Spencer J, Bevan DH, Connolly CE, Kirkham N, et al. Malignant histiocytosis of the intestine: a T cell lymphoma. Lancet 1985 ;ii:688-91.

42 Wright DH, Jones DB, Clark H, Mead GM, Hodges E, Howell WM. Is adult-onset coeliac disease due to a lowgrade lymphoma of intraepithelial T lymphocytes? Lancet 1991;337:1373-4.

43 Alfsen GC, Beiske K, Bell H, Marton PF. Low-grade intestinal lymphoma of intraepithelial $\mathrm{T}$ lymphocytes with concomitant enteropathy-associated T-cell lymphoma: case report suggesting a possible histogenic relationship. Hum Pathol 1989;20:909-13.

44 Howell WM, Leung ST, Jones DB, Nakshabendi I, Hall MA, Lanchbury JS, et al. HLA-DRB, -DQA and -DQB polymorphism in celiac disease and enteropathy-associated T-cell lymphoma: common features and additional risk factors for malignancy. Hum Inmunol 1995; 43:29-37.

45 Ploski R, Ek J, Thorsby E, Sollid LM On the HLADQ $\left(x 1^{*} 0501, \beta 1^{*} 0201\right)$-associated susceptibility in celiac disease: a possible gene dosage effect of DQB $1^{*} 0201$. disease: a possible gene dosage
Tissue Antigens 1993;41:173-7.

46 Congia M, Cucca F, Frau F, Lampis R, Melis L, Clemente $M G$, et al. A gene dosage effect of the DQA ${ }^{*} 0501 /$ $\mathrm{DQB} 1 * 0201$ allelic combination influences the clinical heterogeneity of celiac disease. Hum Immunol 1994;40: 138-42.

47 Fossum B, Breivik J, Meling GI, Gedde-Dahl III T, Hansen $\mathrm{T}$, Knutsen I, et al. A K-ras 13Gly-Asp mutation is recognised by HLA-DQ7 restricted T cells in a patient with colorectal cancer. Modifying effect of $\mathrm{DQ} 7$ on estab costablished 506-11.

48 Poppema S, Visser L. Absence of HLA class I expression by Reed-Sternberg cells. Am $\mathcal{F}$ Pathol 1994;145:37-41.

49 Branch P, Bicknell DC, Rowan A, Bodmer WF, Karran P. Immune surveillance in colorectal carcinoma. Nature Genet 1995;9:231-2. 